\title{
Correction
}

\section{Correction to: Uniqueness of the Representation in Homogeneous Isotropic LQC}

\author{
Jonathan Engle ${ }^{1,2}$, Maximilian Hanusch ${ }^{1}$ (D), Thomas Thiemann ${ }^{2}$ \\ 1 Department of Physics, Florida Atlantic University, 777 Glades Road, Boca Raton, FL 33431, USA. \\ E-mail: jonathan.engle@fau.edu; hanuschm@fau.edu \\ 2 Institute for Quantum Gravity, Friedrich-Alexander University Erlangen-Nürnberg, Staudtstraße 7, \\ 91058 Erlangen, Germany. E-mail: thomas.thiemann@gravity.fau.de
}

Received: 16 April 2018 / Accepted: 29 April 2018

Published online: 30 July 2018 - ( ) Springer-Verlag GmbH Germany, part of Springer Nature 2018

\section{Correction to: Commun. Math. Phys. 354, 231-246 (2017)} https://doi.org/10.1007/s00220-017-2881-2

We correct some oversights contained in [1].

(1) "Campiglia" was accidentally misspelled at several points in the paper. The authors apologize for this.

(2) The authors forgot to provide the definition of the characters [first used in equation (9)]

$$
\chi_{\lambda}: \mathbb{R} \mapsto \mathbb{C}, \quad t \mapsto \mathrm{e}^{\mathrm{i} \lambda t} \quad \forall \lambda \in \mathbb{R} ;
$$

which are the generators of the almost periodic functions $C_{\mathrm{AP}}(\mathbb{R})$ on $\mathbb{R}$.

(3) As noticed in [2], in the continuity part of Sect. 4 it had been overseen that, instead of $v \circ \varphi \in C_{0}(\mathbb{R})$, we have $v \circ \varphi \in \operatorname{span}_{\mathbb{C}}\left(\chi_{0}\right)+C_{0}(\mathbb{R})$ for $\varphi \in C_{0}(\mathbb{R})$ and $\nu:(-1,1) \ni t \mapsto \sqrt{1+t}$. This issue can be fixed, of course, by adding " -1 " in the definition of $\nu$. We then have $\sqrt{1+\varphi} \in \mathfrak{D}$ (instead of $\sqrt{1+\varphi} \in \mathfrak{d}$, as wrongly stated in the paper) - This, however, makes no difference for our conclusion that $1+\omega_{\mathfrak{d}}(\varphi) \geq 0$ holds, because $\omega$ is defined on full $\mathfrak{D}$. The revised argumentationstarting after Eq. (14) on page 238, and ending after the mentioned conclusion-then reads as follows.

\section{Revised argumentation:}

To see this, first observe that the smooth function

$$
v:(-1,1) \ni t \mapsto \sqrt{1+t}-1
$$


(and each of its derivatives) can be represented by a power series. Thus, by completeness of $\overline{\mathfrak{d}}$, for $\varphi \in \overline{\mathfrak{d}}$ real valued with $\|\varphi\|_{\infty}<1$, we have $\nu \circ \varphi \in \overline{\mathfrak{d}}$ as well as

$$
v^{(n)} \circ \varphi \in \operatorname{span}_{\mathbb{C}}\left(\chi_{0}\right)+\overline{\mathfrak{d}} \quad \forall n \geq 1 .
$$

Then, for $\varphi \in \mathfrak{d}$, the chain rule gives

$$
\partial_{t}(\nu \circ \varphi)=(\dot{v} \circ \varphi) \cdot \dot{\varphi} \in \overline{\mathfrak{d}} .
$$

Now, the right hand side is differentiable; and, applying the same arguments inductively, we find that $(\nu \circ \varphi)^{(n)} \in \overline{\mathfrak{d}}$ holds for each $n \in \mathbb{N}$; hence,

$$
\nu \circ \varphi=\sqrt{1+\varphi}-\chi_{0} \in \mathfrak{d} .
$$

We thus have $\varphi_{v}:=\sqrt{1+\varphi} \in \mathfrak{D}$ with

$$
1+\omega_{\mathfrak{d}}(\varphi)=\omega(\mathbb{1}+\widehat{\varphi})=\omega\left(\widehat{\varphi}_{\nu}^{*} \widehat{\varphi}_{\nu}\right) \geq 0 .
$$

(4) In the equations (24) and (25), the $\gamma$-factor was misplaced. More specifically,

- Equation (24) must read

$$
\sum_{i=1}^{3} \frac{V_{0}[\mathcal{C}]}{k \gamma} \frac{\left|v_{1} v_{2} v_{3}\right|}{v_{i}} \frac{\mathrm{d}}{\mathrm{d} x^{\mathbf{0}}}\left(\gamma \omega_{\mathbf{i}}^{i 0}-\frac{1}{2} \epsilon^{i j k} \omega_{\mathbf{i} j k}\right) .
$$

- Equation (25) must read

$$
c^{i}:=\gamma \omega_{\mathbf{i}}^{i 0}-\frac{1}{2} \epsilon^{i j k} \omega_{\mathbf{i} j k}, \quad p_{i}:=\frac{V_{0}[\mathcal{C}]}{k \gamma} \frac{\left|v_{1} v_{2} v_{3}\right|}{v_{i}} .
$$

Acknowledgements We thank Christian Fleischhack for communicating to us [2] the technical oversight mentioned in 3 .

\section{References}

1. Engle, J., Hanusch, M., Thiemann, Th.: Uniqueness of the representation in homogeneous isotropic LQC. Commun. Math. Phys. 354, 231-246 (2017)

2. Fleischhack, Ch.: Continuity of States on Non-Unital Differential Algebras in Loop Quantum Cosmology. e-print: arXiv:1803.08944 [math-ph] 